\title{
Vehicle Routing Problem for Fashion Supply Chains with Cross-Docking
}

\author{
Zhi-Hua Hu, ${ }^{1,2}$ Yingxue Zhao, ${ }^{3}$ and Tsan-Ming Choi ${ }^{4}$ \\ ${ }^{1}$ School of Economics and Management, Tongji University, Shanghai 200092, China \\ ${ }^{2}$ Logistics Research Center, Shanghai Maritime University, Shanghai 200135, China \\ ${ }^{3}$ School of International Trade and Economics, University of International Business and Economics, Beijing 100029, China \\ ${ }^{4}$ Institute of Textiles and Clothing, The Hong Kong Polytechnic University, Hung Hom, Kowloon, Hong Kong
}

Correspondence should be addressed to Yingxue Zhao; zyx@amss.ac.cn

Received 7 December 2012; Accepted 17 January 2013

Academic Editor: Pui-Sze Chow

Copyright (C) 2013 Zhi-Hua Hu et al. This is an open access article distributed under the Creative Commons Attribution License, which permits unrestricted use, distribution, and reproduction in any medium, provided the original work is properly cited.

\begin{abstract}
Cross-docking, as a strategy to reduce lead time and enhance the efficiency of the fashion supply chain, has attracted substantial attention from both the academy and the industry. Cross-docking is a critical part of many fashion and textiles supply chains in practice because it can help to achieve many supply chain strategies such as postponement. We consider a model where there are multiple suppliers and customers in a single cross-docking center. With such a model setting, the issue concerning the coordinated routing between the inbound and outbound routes is much more complex than many traditional vehicle routing problems (VRPs). We formulate the optimal route selection problems from the suppliers to the cross-docking center and from the cross-docking center to the customers as the respective VRPs. Based on the relationships between the suppliers and the customers, we integrate the two VRP models to optimize the overall traveling time, distance, and waiting time at the cross-docking center. In addition, we propose a novel mixed 0/1 integer linear programming model by which the complexity of the problem can be reduced significantly. As demonstrated by the simulation analysis, our proposed model can be solved very efficiently by a commonly used optimization software package.
\end{abstract}

\section{Introduction}

The fashion industry's supply chain is full of uncertainty, unpredictability, and various complexities, as studied by Lo et al. [1]. The common characteristics of the fashion supply chains include short product life cycle, high product variety, high demand volatility with low predictability [2], long and relatively inflexible supply processes, and high impulse purchasing behavior of consumers. Undoubtedly, the supply chain in the fashion industry is complex. As a result, various measures, such as the quick response systems, have been widely adopted in the fashion industry for achieving efficient supply chain management. However, the original functions of quick response system cannot completely overcome the challenges in the fashion supply chain. From the perspective of agile supply chain management, cross-docking (CD) becomes a useful and effective way to speed up the turnaround time of fashion products and decrease the timerelated risks in the supply chain.
In fact, $\mathrm{CD}$ is a popular logistical strategy by which packages of products are unloaded from the inbound vehicle and then are almost directly uploaded into the outbound vehicle with little or no storage in between [3]. As a practical realworld industrial practice, $\mathrm{CD}$ has attracted substantial attention from both the academy and the industry. To implement $\mathrm{CD}$, there is usually a cross-docking center (CDC). Under $\mathrm{CD}$, in many cases, loads are divided into units based on their respective destinations and then are reloaded into the corresponding outbound trucks. This strategy has several potential merits. For example, it can reduce total inventory costs and lead time, enhance customer service level, and improve the relationships with suppliers $[4,5]$.

However, implementing CD requires careful considerations. As Van Belle et al. [3], Schaffer [5], and Choi [6] point out, a couple of factors are critical to the success of $\mathrm{CD}$ operations. First, since CD requires support from the supplier side, it requires the supplier to possess the capability to 


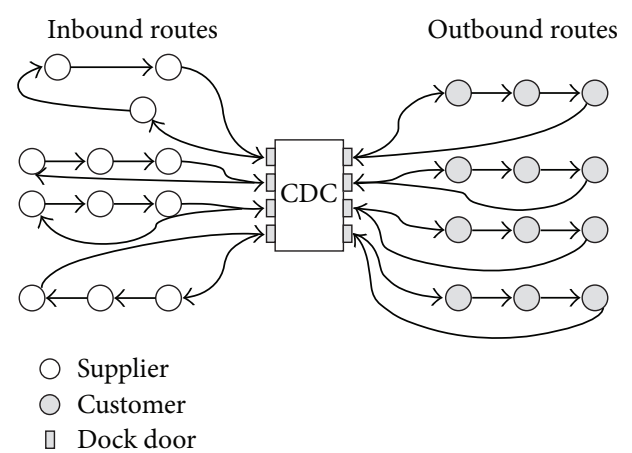

FIGURE 1: Inbound and outbound routes merging at CDC.

respond reliably and quickly within a short lead time. Second, CD schedule depends heavily on the availability of accurate information about the arrival times, the departure times, and the destinations that products will be delivered to. As a result, just-in-time information sharing is necessary for successful implementation of $\mathrm{CD}$. Third, the precision on time and space is critical for exercising CD. To be specific, short lead time must be ensured by the very accurate arrival and departure of goods. Thus, the corresponding transport system must have the right capability to prevent delays. Fourth, transport process should be simple enough to reduce the uncertainty inherent in it. It will be most desirable if the demands are reasonably big and can be well predicted because the resulting transport volume can be increased by merging multiple orders.

Figure 1 illustrates how the inbound and the outbound routes are merged at a typical CDC for fashion products. In Figure 1, it is presumed that all trucks start and arrive CDC finally. In each route, a truck visits a series of suppliers and customers to pickup and deliver goods. According to the specific supply relationship between the suppliers and the customers, at CDC goods are unloaded from the inbound trucks and are then loaded onto the outbound trucks that serve the target customers. In practice, each route should only visit a little set of suppliers or customers; otherwise, efficiency will be reduced by the frequent unloading/loading processes at CDC.

It is obvious that with the $\mathrm{CDC}$, even though the inbound and the outbound routes can be modeled, respectively, as two independent VRPs, a system-wide optimal performance requires the coordination between the unloading task of the inbound trucks and the uploading task of the outbound trucks, which arises a question as how to achieve this coordination. In this paper, we will devote to addressing this issue. To be specific, based on the relationships between the suppliers and the customers, we integrate the two independent VRP models to develop the strategies that can optimize the overall traveling time, distance, and waiting time at the CDC. In addition, we propose a novel mixed 0/1 integer linear programming model that can significantly reduce the complexity of the problem under study. As a matter of fact, as demonstrated by the simulation analysis, our proposed model can be solved very efficiently by a commonly used optimization software package.
The rest of this paper is organized as follows. In Section 2, we present a concise review on the relevant literature. In Section 3, we describe the problem under study and present the assumptions used. In Section 4, we construct the analytical optimization model. We conduct the simulation analysis in Section 5. We conclude the study in Section 6.

\section{The Literature Review}

The fashion supply chain is full of uncertainty and unpredictability. It is in general a rather complex system and requires very careful analysis in order to find rooms to improve its efficiency. As studied by Lo et al. [1], quick response systems have been rather broadly used in the fashion industry to cut the lead time and respond fast to market changes. Lo et al. [1] explore how an intelligent system can effectively capture the requirements of customers and help manage the supply chain by a multiagent system approach. Choi $[6,7]$ argue that the biggest challenge in fashion supply chain management comes from the uncertain demand of customers, which leads to big supply-demand mismatch problems of having over- and understocking. He believes that a quick response system is a probable measure to dampen these problems (see Choi and Sethi [8] for a comprehensive review of quick response systems). Bertolini et al. [9] suggest that leaders in the fashion industry have the common features of putting emphasis on fashion brand management, networked production, chain store distribution, and committed investments in up-to-date logistics and supply chain management information systems. Salmeron and Hurtado [10] think that the fashion industry can employ different channels, such as business-to-customer (B2C) and electronic commerce (EC), to catch customers. They hence suggest the EC-based logistics scheme for helping fashion supply chain management. Guercini and Runfola [11] present different theoretical perspectives on the issue of business networks and their role in the process of internationalization. They conduct a case study based on a vertically integrated fashion firm which has implemented branding and retailing strategies in an international context. Christopher et al. [12] and Şen [13] show that the characteristics of fashion supply chains include short product life cycle and high demand volatility with low predictability. The fashion supply chains also involve many inflexible processes. These characteristics are also partially identified by Bruce et al. [14]. Based on these inherent features of fashion supply chains, there are proposals on using agile SCM approaches with a goal of achieving quick response to market demand changes.

To achieve agile SCM, the use of information systems is a popular measure. In a study regarding fashion retailers in the UK, Birtwistle et al. [15] find through an empirical survey that information technology is particularly important to enhance communication in supply chains with multiple large private label fashion retailers. They also believe that these fashion retailers have not yet fully understood the benefits of implementing a quick response strategy on the internal supply chain management rather than on an external supply chain strategy. In setting up the quick response program for 
fashion products, it is beneficial to speed up the turnaround process by employing $\mathrm{CD}$ technology.

The previous research on CD generally focuses on the following issues: (1) location decision of CDC, (2) layout design of dock, (3) synchronized inbound and outbound truck assignment, (4) routing problem of load units in dock, and (5) routing problem for inbound and outbound operations. We review concisely some representative papers in the following (notice that some prior studies are devoted to addressing a certain aspect of these decision problems and some take an integrated approach which take into account and try to find the system-wide optimal solutions. For more, refer to Van Belle et al. [3]).

2.1. Location Decision of CDC. To implement CD, the presence of CDC is critical, and its location is very influential. In fact, CDC helps coordinate suppliers and customers with distances among them. By consolidating the goods from different suppliers to a specific set of customers, the logistics costs can be reduced which helps achieve the optimal supply chain performance. In the literature, the majority of models related to this issue focus on the location problem with distribution centers. For example, Ross and Jayaraman [16] propose an evaluation model with new heuristic procedures for deciding the optimal location of cross-docks and distribution centers in the design of supply chain network. Their model involves multiple product families, a central manufacturing plant site, multiple CD and distribution center sites, and retail outlets facing multiple demand units of several commodities. By utilizing the simulated annealing methodology, they describe two heuristics that can be used to find the "near globally optimal" strategies for the design and employment of distribution system. Recently, Sung and Yang [17] design a novel algorithm to optimally locate CDC and allocate the respective vehicles. They model the problem under study as a set-partitioning-based problem and derive efficient procedure to identify the optimal solution.

2.2. Layout Design of Dock. In the literature, most prior studies on warehouse layout design of warehouses are closely related to the dock layout design. In fact, dock layout design problem generally includes several subproblems, such as the optimization of the quantity and location of facilities and the prediction of the frequency and strength of facility employments. Heragu et al. [18] divide warehouse into several functional areas, including reserve storage area, forward (order collation) area, and CD. Using data available to a warehouse manager, they develop a mathematical model and a heuristics algorithm that can be used to find the jointly optimal solution for the allocation of products to these functional areas and the size of each functional area. Later on, Alpan et al. [19] consider an analytical model in a multiple receiving and shipping doors cross-docking environment. They develop the optimal or near optimal facility scheduling policies that can minimize the total costs with respect to the transshipment operations at the facility for cross-docking. Most recently, Vis and Roodbergen [20] present a dynamic optimal design methodology to select control policies which help to determine the optimal layout rules for cross-docking facilities.

2.3. Synchronized Inbound and Outbound Truck Assignment. It is well known that CDCs have two typical functions: temporary storage space and consolidation of goods from trucks to trucks. However, the storage space of CDC is commonly limited. As a norm, goods should not be kept in docks for longer than 24 hours. Therefore, the main function of CDC is to consolidate goods from the inbound trucks to the outbound trucks. In order to improve the efficiency of truck usage, synchronizing the respective loading operations is a critical problem. In the literature, Soltani and Sadjadi [21] propose a hybrid simulated annealing and hybrid variable neighborhood searching algorithm to solve the cross-docking problem with a goal of achieving the best sequence of truck pairs. Boysen [22] examines a typical truck assignment model in the context of $\mathrm{CD}$, where zero inventory is assumed based on the features of supply chains in food transportation. Boysen et al. [23] design a model that deals with the coordination problem between the inbound and outbound trucks. They propose some efficient methods to tackle the problem. After that, Vahdani and Zandieh [24] and Lee et al. [25] solve the truck assignment problem by some new heuristic approaches. McWilliams [26] proposes a dynamic load-balancing algorithm to solve the parcel hub-scheduling problem, which is known as a combinatorial optimization problem. The problem involves a large number of inbound trailers but a much smaller number of unload docks. The parcels in the inbound trailers must be unloaded, sorted and transferred to the load docks, and then loaded onto the outbound trailers. Because the transfer operation is laborintensive and operates in a time-sensitive environment, the loading operations of the parcels must be completed in a way to minimize the time span of the transfer operation. Oh et al. [27] address the operational problems of the cross-docking system in a mail distribution center. The center has two types of doors, receiving doors and shipping doors. The assignment of destinations to shipping doors, clustering of destinations to form groups, and determination of the number of groups are major operational problems directly related with the efficiency of the center. They develop a nonlinear optimization model and employ two methods, namely, the three-phase heuristic procedure and the genetic algorithm, to derive the optimal solution. Recently, Yu and Egbelu [28] consider the truck assignment problem that deals with temporary inventory when scheduling the assignment of truck to door. By cross-docking, they find that the turnaround times for customer orders will be reduced.

2.4. Routing Problem of Load Units in Dock. Unlike many traditional warehousing "storage-based" operations, the efficiency of space and facility usage is an important criterion to assess cross-docking operations. Routing problem on loading units in dock usually focuses on these objectives. Cohen and Keren [29] study the assignment model that determines the amount of freight handling within the cross-dock facility with higher efficiency. Due to the immense complexity, they solve 
their dock-door assignment problem by using heuristics. Recently, Vis and Roodbergen [4] propose a solution for goods positioning problem in docks with an objective to minimize the routing time. They model this problem as an application of the minimum cost flow problem and show the applicability of the model for different types of layouts and priorities.

\subsection{Routing Problem for Inbound and Outbound Operations.} Transportation from suppliers to CDC and from CDC to customers requires careful planning. In fact, how to synchronize the inbound and outbound routes is a difficult problem. In the literature, this type of problem can be modeled as a coordination problem with multiple VRPs. Vahdani and Zandieh [24] apply five metaheuristic algorithms to schedule the trucks in cross-dock systems such that the total operational time is minimized when a temporary storage buffer to hold items is located at the shipping dock. Lee et al. [25] design an integrated model with the consideration of both cross-docking and route scheduling. They propose a heuristic algorithm based on a tabu search method to solve the problem. Notice that the vehicle routing problem with cross-docking (VRPCD) usually focuses on fulfilling a set of transportation requests using a set of vehicles to accomplish the pickup and delivery tasks [30]. Between those operations, there is an important consolidation process at the crossdocking centre. Alpan et al. [31] study the transshipment scheduling problem in a multiple inbound and outbound dock setting. They identify the best schedule of transshipment operations with a goal of minimizing the total inventory holding and truck replacement costs. Wen et al. [32] investigate the VRPCD with an objective to minimize the total travel time under the given time window constraints at the nodes. They formulate the problem as a mixed integer program and develop a tabu search heuristic embedded within an adaptive memory procedure to solve the problem. Liao et al. [33] study the inbound and outbound truck sequencing for cross-docking operations with the goal of minimizing total operation time.

As reviewed above, cross-docking-related optimization problems are widely explored. In fact, VRP is also a widely explored topic (e.g., see the reviews by Eksioglu et al. [34], Pillac et al. [35], and Drexl [36]). However, almost all the studies on VRP in the literature focus on scheduling the optimal route plans in a run to satisfy the constraints on cost, time, and other resource limitations. The research on the coordination between two VRP models is rarely seen in the literature, which also indicates the major difference between this paper and the current literature.

\section{Problem Description}

The scenario examined in this paper is depicted in Figure 1. To be specific, this paper first studies (i) scheduling VRPs on both the inbound and outbound operations for CDC with fashion products and (ii) the optimal assignment of routes to dock doors. After that, this paper explores the coordination challenge between two VRP models. In our problem, the following inputs are identified. First, the suppliers and the customers involved in the plan are provided as well as the available trucks to pickup and deliver goods. Second, the supply relationship between suppliers and customers is provided in a form where the amount that each supplier supplies to any customer is filled. Third, the traveling time and capacity of each truck are specified, and the respective upper bounds are provided. The scheduling problem for all routes to serve the suppliers or customers can be formulated apparently as a VRP. The optimal solution will guarantee that the traveling time and involved trucks are minimized.

In order to facilitate the formulation and provide solutions to the key problems, some presumptions are summarized as follows.

(1) All trucks are waiting at CDC. When they finish the pickup or delivery tasks, they return to CDC. Therefore, all scheduled routes are close, beginning, and ending at CDC.

(2) The capability of CDC is always adequate.

(3) The time windows at suppliers and customers are all not considered.

(4) The cost of VRP is measured only by traveling time.

(5) In the planning horizon, each truck can only serve one route at a time.

\section{Model Formulation}

4.1. Indices and Parameters. In the following, we first present the indices and parameters used for the outbound VRP and for the inbound VRP. The indices and parameters can be defined in a similar way as follows.

(1) $\mathrm{ON}=\{0,1,2, \ldots, \mathrm{ONN}\}$ : the indices for the customers. The first, 0 , represents the CDC. ONN is the number of customers.

(2) $\mathrm{OV}=\{1,2, \ldots, \mathrm{OVN}\}$ : the indices for the available vehicles. In other words, it is for the routes. OVN is the number of vehicles.

Similarly, IN and IV are defined for the inbound VRP.

(3) $\operatorname{IOS}_{i, o}$ defines the amount of products transported from the supplier $i \in \mathrm{IN}$ to the customer $o \in \mathrm{ON}$.

(4) $\operatorname{IOSB}_{i, o} \in\{0,1\}$ is a Boolean variable to indicate the supply relationship between the supplier $i \in \mathrm{IN}$ to the customer $o \in \mathrm{ON}$. It can be computed by

$$
\operatorname{IOSB}_{i, o}= \begin{cases}1, & \mathrm{IOS}_{i, o}>0 \\ 0, & \text { else. }\end{cases}
$$

(5) $\mathrm{OR}_{o}$ : the demand of customer $o \in \mathrm{ON}$. It can be computed from the parameter $\operatorname{IOS}_{i, o}$ by (2). Similarly, $\mathrm{IR}_{i}$ can be defined and computed by (3) as follows:

$$
\begin{aligned}
& \mathrm{OR}_{o}=\sum_{i \in \mathrm{IN}} \mathrm{IOS}_{i, o}, \\
& \mathrm{IR}_{i}=\sum_{o \in \mathrm{ONN}} \mathrm{IOS}_{i, o} .
\end{aligned}
$$


(6) $\mathrm{ORMIN}_{i}=\min _{j \neq i}\left\{\mathrm{OR}_{j}\right\}$ is defined to avoid the subtours in the outbound VRP. Similarly, $\operatorname{IRMIN}_{i}=$ $\min _{j \neq i}\left\{\operatorname{IR}_{j}\right\}$ is defined to avoid the subtours in the inbound VRP.

(7) $\mathrm{OC}_{i, j}$ : the cost associated with traveling from customer $i \epsilon \mathrm{ON}$ to customer $j \in \mathrm{ON}$. In this study, it is the traveling time.

(8) OQ defines the capacity of all vehicles.

(9) $\mathrm{OL}_{v}$ defines the upper limit of the traveling time of the vehicle $v \in \mathrm{OV}$.

$\mathrm{IC}_{i, j}, \mathrm{IQ}$, and $\mathrm{IL}_{v}$ are defined similarly for the inbound VRP.

\subsection{Decision Variables}

(1) $o x_{i, j, v} \in\{0,1\}: o x_{i, j, v}=1$ indicates that the customers $i \epsilon \mathrm{ON}$ and $j \epsilon \mathrm{ON}$ are served by the vehicle $v \in \mathrm{OV}$ sequentially in a single route without another customer served between them.

(2) $o y_{i, v} \in\{0,1\}: o y_{i, v}=1$ indicates that the customer $i \in O N$ is served by the vehicle $v \in \mathrm{OV}$.

(3) $o z_{v} \in\{0,1\}: o z_{v}=1$ denotes that the vehicle $v \in \mathrm{OV}$ is used, and it serves at least one customer.

(4) $o u_{i}: o u_{i}$ defines the unloaded amount from the vehicle just after leaving the customer $i \in \mathrm{ON}$. This variable is used to avoid the subtours in a route in the results of VRP.

$o x_{i, j, v} \in\{0,1\}, o y_{i, v} \in\{0,1\}, o z_{v} \in\{0,1\}$, and $o u_{i}$ are defined in a same way for the inbound VRP.

(5) $r_{i v, o v} \in\{0,1\}:$ if $r_{i v, o v}=1$, outbound vehicle ov $\epsilon \mathrm{OV}$ must wait for $i v \in \mathrm{IV}$ because at least one served customer requires products from at least one supplier by $i v \epsilon \mathrm{OV}$.

The first three matrices are to represent the inbound and outbound VRPs. The fourth is used to avoid the subtours in a route. The last variable is defined to represent the measure that the outbound trucks will wait for the inbound trucks.

4.3. Objectives. The objectives considered in the model can be classified into two types. The first is to optimize the inbound and outbound VRPs. The second is to minimize the waiting time of the outbound trucks for the inbound trucks.

(1) The cost of VRP should be minimized as defined in (4) for the inbound and outbound VRPs:

$$
\begin{aligned}
& \text { minimize: } i v r p c=\sum_{i \in \mathrm{IN}, i \in \mathrm{IN}, v \in \mathrm{IV}}\left(\mathrm{IC}_{i j} \cdot i x_{i j v}\right), \\
& \text { minimize: } o v r p c=\sum_{i \in \mathrm{ON}, j \in \mathrm{ON}, v \in \mathrm{OV}}\left(\mathrm{OC}_{i j} \cdot o x_{i j v}\right) .
\end{aligned}
$$

(2) The number of utilized trucks should be minimized as defined in

$$
\begin{aligned}
& \text { minimize: } i z s=\sum_{v \in \mathrm{IV}} i x_{v}, \\
& \text { minimize: } o z s=\sum_{o \in \mathrm{OV}} i x_{v} .
\end{aligned}
$$

(3) The waiting time of the outbound trucks for the inbound trucks should be minimized with respect to the corresponding numbers of inbound routes for all outbound routes:

$$
\text { minimize: } w=\sum_{o v \in \mathrm{OV}, i v \in \mathrm{IV}} r_{i v, o v} \text {. }
$$

With the above five objectives, the problem under study should be formulated as a multiobjective optimization model.

4.4. Constraints. The first series of constraints is order to ensure that the requirements of capitalized VRP are fulfilled. We first formulate the constraints for the outbound VRP as follows, and the constraints for the inbound VRP can be formulated in a similar way:

(1) the load must be less than the vehicle capacity, as shown in

$$
\forall v \in \mathrm{OV}, \quad \sum_{i \in \mathrm{ON} \backslash\{0\}}\left(\mathrm{OR}_{i} \cdot o y_{i, v}\right) \leq \mathrm{OQ}
$$

(2) each customer must be served by one and only one route, as defined in

$$
\forall i \epsilon \mathrm{ON} \backslash\{0\}, \quad \sum_{v \in \mathrm{OV}} o y_{i, v}=1
$$

(3) if the customer $j \in \mathrm{ON}$ is delivered by the vehicle $v \in \mathrm{OV}$, at least one $v \in \mathrm{OV}$ must reach $j \epsilon \mathrm{ON}$ from one $i \in \mathrm{ON}$, which can be formulated as

$$
\forall j \in \mathrm{ON} \backslash\{0\}, v \in \mathrm{OV}, \quad \sum_{i \in \mathrm{ON}, i \neq j} o x_{i, j, v}=o y_{j, v} \text {, }
$$

(4) if $i \epsilon \mathrm{ON}$ is delivered by $v \in \mathrm{OV}$, after that it must reach one $j \in \mathrm{ON}$ :

$$
\forall i \in \mathrm{ON} \backslash\{0\}, v \in \mathrm{OV}, \quad \sum_{j \in \mathrm{ON}, j \neq i} o x_{i, j, v}=o y_{i, v},
$$

the above two constraints are order to ensure the connectivity of a route,

(5) cost constraint is defined in

$$
\forall v \in \mathrm{OV}, \quad \sum_{i \in \mathrm{ON}, j \in \mathrm{ON}}\left(\mathrm{OC}_{i j} \cdot o x_{i j v}\right) \leq \mathrm{OL}_{v},
$$


(6) the variable of vehicle usage can be defined by the customer-vehicle assignment variables, which leads to the constraint defined in

$$
\begin{aligned}
& \forall v \in \mathrm{OV}: \\
& \left\{\begin{array}{l}
o z_{v} \geq \mathrm{MIND} \cdot \sum_{i \in \mathrm{ON} \backslash\{0\}} o y_{i v} \\
o z_{v} \leq \sum_{i \in \mathrm{ON} \backslash\{0\}} o y_{i v} \\
\sum_{j \in N \backslash\{0\}} x_{0 j v}=o z_{v} \\
\sum_{i \in \mathrm{ON} \backslash\{0\}} o x_{i 0 v}=o z_{v},
\end{array}\right.
\end{aligned}
$$

given that the relationships among $o x, o y$, and $o z$ should be modeled with the consistence among them, constraints defined in (13) are required to satisfy

$$
\begin{gathered}
\sum_{i \in \mathrm{ON} \backslash\{0\}, v \in \mathrm{OV}} o x_{0 i v}=\sum_{v \in \mathrm{OV}} o z_{v}, \\
\sum_{i \in \mathrm{ON} \backslash\{0\}, v \in \mathrm{OV}} o x_{i 0 v}=\sum_{v \in \mathrm{OV}} o z_{v}, \\
\forall i \in \mathrm{ON} \backslash\{0\}, \quad \sum_{j \in \mathrm{ON}, v \in \mathrm{OV}} o x_{i j v}=1, \\
\forall j \in \mathrm{ON} \backslash\{0\}, \quad \sum_{i \in \mathrm{ON}, v \in \mathrm{OV}} o x_{i j v}=1,
\end{gathered}
$$

(7) the above constraints can ensure the connectivity of each route, but not avoid the subtours. Hence, the following linear constraints in (14) are given to restrict the subtours

$$
\begin{aligned}
& \forall i \in \mathrm{ON} \backslash\{0\}, \\
& o u_{i}+\left(Q-\mathrm{ODMIN}_{i}-\mathrm{OR}_{i}\right) \cdot o x_{0 i}-\mathrm{ODMIN}_{i} \cdot o x_{i 0} \\
& \leq Q-\mathrm{ODMIN}_{i} \\
& \forall i \in \mathrm{ON} \backslash\{0\} \\
& o u_{i}+\mathrm{ODMIN}_{i} \cdot o x_{0 i}+\left(\mathrm{OR}_{i}+\mathrm{ODMIN}_{i}\right) \cdot o x_{i 0} \\
& \geq \mathrm{OR}_{i}+\mathrm{ODMIN}_{i} \\
& \forall i, j \in \mathrm{ON} \backslash\{0\}, \quad i \neq j, \\
& o u_{i}-o u_{j}+Q \cdot o x_{i j}+\left(Q-\mathrm{OR}_{i}-\mathrm{OR}_{j}\right) \cdot o x_{j i} \leq \mathrm{Q}-\mathrm{OR}_{j} \text {. }
\end{aligned}
$$

The second series of constraints deal with the definition of $r$, which is used to represent the waiting time of the outbound trucks for the inbound trucks. If $r_{i v, o v}=1$, at least one customer in the outbound route is supplied by the supplier
TABLE 1: Basic parameter settings of the case demonstration.

\begin{tabular}{lccc}
\hline Parameter & INN & IVN & IQ \\
Value & 8 & 4 & 40 \\
\hline Parameter & ONN & OVN & OQ \\
Value & 8 & 4 & 40 \\
\hline
\end{tabular}

in the inbound route. This relation can be formulated as (15). It can be further expressed by a linear expression as shown in

$$
\begin{aligned}
& \left(r_{i v, o v}=1\right) \longleftrightarrow\left\{\begin{array}{l}
\exists i \in \mathrm{IN}, \exists o \in \mathrm{ON}: \mathrm{ISOB}_{i, o}=1, \\
i y_{i, i v}=1, \\
o y_{o, o v}=1,
\end{array}\right. \\
& \forall i v \in \mathrm{IV}, \quad \text { ov } \epsilon \mathrm{OV}, \quad i \in \mathrm{IN}, \quad o \in \mathrm{OV}: r_{i v, o v} \\
& \geq \mathrm{ISOB}_{i, o}+i y_{i, i v}+o y_{o, o v}-2 .
\end{aligned}
$$

The model of coordinated VRP for CD is built upon the above indices, parameters, decision variables, objectives, and constraints. All decision variables are 0/1 integers. All analytical expressions are given in linear integer formats. Therefore, the proposed model is a multiobjective integer linear programming model.

\section{Simulation Analysis}

As discussed in Section 4, the proposed model is a multiobjective integer linear programming model. In the following analysis, Xpress-IVE module [37] is employed as the mixed integer linear programming (MILP) solver, which is installed in a PC with Pentium $2.4 \mathrm{GHz}$ processor and $2 \mathrm{G}$ RAM.

The analysis is conducted in two steps. First, a case is demonstrated. Second, the model is solved with different parameter settings. Because the model is multiobjective, it will be transferred into a single objective model by adjusting the other objectives to be constraints. With the five objectives, the minimization of used trucks can be restricted by constraints conveniently. First, the numbers of used trucks for inbound or outbound will not be large. Second, the numbers can be adjusted by setting the upper bounds. Therefore, in the following, these two objectives are taken as constraints by adjusting IVN and OVN. Another two objectives are to minimize the costs of inbound and outbound VRP routes. The fifth objective is to minimize the waiting time $w$ as defined in (6).

5.1. Case Demonstration. In this part, the input parameters are given for a small-scale problem. In Table 1, the basic parameters, including the serviced node numbers, the trucks, and their capacities of the inbound and outbound VRPs, are given. In Tables 2 and 3, the cost matrices of the transport networks for inbound and outbound VRPs are detailed. In Table 4, the supply matrix between suppliers and customers is provided. Each supplier will supply to two customers. In the following, by adjusting the objective function and decomposing it as different subobjectives, the model can be solved to yield the optimal plans of distribution and consolidation. 
TABLE 2: Cost matrix of the inbound VRP.

\begin{tabular}{cccccccccc}
\hline & 0 & 1 & 2 & 3 & 4 & 5 & 6 & 7 & 8 \\
\hline 0 & 0 & 3 & 10 & 3 & 6 & 9 & 9 & 10 & 4 \\
1 & 3 & 0 & 10 & 7 & 7 & 10 & 5 & 5 & 1 \\
2 & 10 & 10 & 0 & 2 & 7 & 6 & 3 & 2 & 6 \\
3 & 3 & 7 & 2 & 0 & 6 & 2 & 7 & 4 & 8 \\
4 & 6 & 7 & 7 & 6 & 0 & 9 & 1 & 9 & 10 \\
5 & 9 & 10 & 6 & 2 & 9 & 0 & 10 & 1 & 3 \\
6 & 9 & 5 & 3 & 7 & 1 & 10 & 0 & 8 & 10 \\
7 & 10 & 5 & 2 & 4 & 9 & 1 & 8 & 0 & 5 \\
8 & 4 & 1 & 6 & 8 & 10 & 3 & 10 & 5 & 0 \\
\hline
\end{tabular}

TABLE 3: Cost matrix of the outbound VRP.

\begin{tabular}{cccccccccc}
\hline & 0 & 1 & 2 & 3 & 4 & 5 & 6 & 7 & 8 \\
\hline 0 & 0 & 3 & 5 & 1 & 10 & 9 & 1 & 7 & 10 \\
1 & 3 & 0 & 7 & 5 & 1 & 4 & 2 & 9 & 3 \\
2 & 5 & 7 & 0 & 3 & 9 & 4 & 9 & 9 & 6 \\
3 & 1 & 5 & 3 & 0 & 8 & 1 & 2 & 3 & 1 \\
4 & 10 & 1 & 9 & 8 & 0 & 2 & 4 & 6 & 2 \\
5 & 9 & 4 & 4 & 1 & 2 & 0 & 6 & 5 & 7 \\
6 & 1 & 2 & 9 & 2 & 4 & 6 & 0 & 8 & 4 \\
7 & 7 & 9 & 9 & 3 & 6 & 5 & 8 & 0 & 1 \\
8 & 10 & 3 & 6 & 1 & 2 & 7 & 4 & 1 & 0 \\
\hline
\end{tabular}

TABLE 4: Supply matrix between suppliers and customers.

\begin{tabular}{llllllllll}
\hline & 0 & 1 & 2 & 3 & 4 & 5 & 6 & 7 & 8 \\
0 & 0 & 0 & 0 & 0 & 0 & 0 & 0 & 0 & 0 \\
\hline 1 & 0 & 5 & 8 & 0 & 0 & 0 & 0 & 0 & 0 \\
2 & 0 & 0 & 4 & 8 & 0 & 0 & 0 & 0 & 0 \\
3 & 0 & 0 & 0 & 6 & 8 & 0 & 0 & 0 & 0 \\
4 & 0 & 0 & 0 & 0 & 6 & 4 & 0 & 0 & 0 \\
5 & 0 & 0 & 0 & 0 & 0 & 5 & 8 & 0 & 0 \\
6 & 0 & 0 & 0 & 0 & 0 & 0 & 5 & 6 & 0 \\
7 & 0 & 0 & 0 & 0 & 0 & 0 & 0 & 7 & 0 \\
8 & 0 & 8 & 0 & 0 & 0 & 0 & 0 & 0 & 4 \\
\hline
\end{tabular}

By aggregating the three objectives by a weight vector $W=\left\{W_{\text {ivrp }}, W_{\text {ovrp }}, W_{w}\right\}$, the multiple objectives, ivrpc, ovrpv, and $w$, are transferred to a single objective, as shown in

$$
\text { minimize: } o b j=W_{i v r p} \cdot i v r p c+W_{\text {ovrp }} \cdot o v r p c+W_{w} \cdot w \text {. }
$$

Xpress-IVE produces optimal solutions corresponding to different settings of the weight vector. The weight vectors and objective values are shown in Table 5. Although the objectives on used trucks are not embedded in the final objective function, in the optimal solution, the trucks are not fully employed in the inbound and outbound VRPs. By the rules in multiobjective decision making, the Pareto optimal set is obtained, which consists of "case 1" and "case 18". In Figures 2 and 3, the VRP plans including the routes with passing suppliers or customers of these two optimal cases are shown. The two cases all use three inbound trucks and three

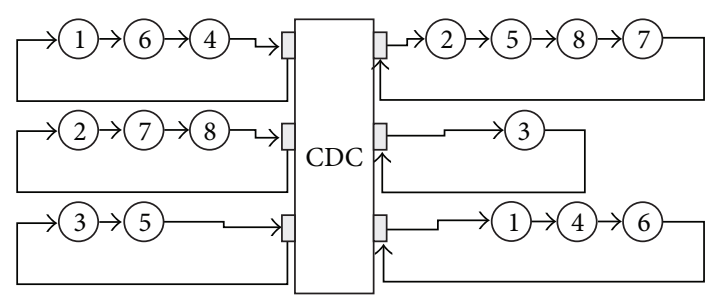

FIgURE 2: VRP plans of the "case 1".

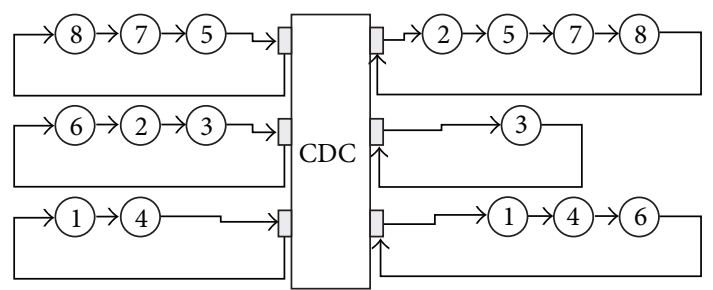

FIGURE 3: VRP plans of the "case 18".

outbound trucks. The routing cost of "case 1 " is 85 , less than 86 in "case 18" at the expense of a higher "waiting time" of 1. Upon these five objectives, other cases are inferior to the two optimal solutions.

5.2. Performance Evaluation. The model deals with two VRPs and the coordination between them. Therefore, one may believe that the corresponding optimization problem cannot be solved with an acceptably good performance because VRP is itself a notorious problem in terms of computational efficiency in mathematical programming. However, in the application of cross-docking, the two VRPs only deal with little-scale or medium-scale problems. The scheduled trucks, passed nodes, and node numbers of routes are all limited. Therefore, it is probable to obtain a promising performance to solve the problem. In order to reveal whether the model can be applied in real-world CDCs, a series of cases generated randomly are solved, and the computation times are recorded. For each setting with the involved inbound and outbound nodes and trucks, the test is repeated for 30 times with different cost matrices and supply matrices. The average computational time is recorded in Table 6. For all 12 cases, the optimal solutions can be obtained. When the scale is increased, the computation time climbs up quickly. According to the industrial observation in China, most CDCs support the applications of six to ten suppliers, four to eight customers, and about four trucks of inbound and outbound transports in an 8-hour plan. The optimal schedules of such a scale can be computed within 30 seconds, which is acceptable. The greater scale of even more than 20 passed nodes and 10 trucks can be solved in 15 minutes. For an 8-hour plan, it is still applicable in real-world CDCs.

\section{Conclusions}

Logistical efficiency plays an increasingly important role in shortening the lead time for an agile fashion supply 
TABLE 5: Optimal objective values of the demonstrative case.

\begin{tabular}{|c|c|c|c|c|c|c|c|c|c|}
\hline Case & $W$ & ivrpc & ovrpc & izs & izs & $w$ & obj & Is it optimal? & Computation time/s \\
\hline 1 & {$[0.1,0.1,0.8]$} & 3 & 3 & 50 & 35 & 8 & 14.9 & $\mathbf{Y}$ & 0.4 \\
\hline 2 & {$[0.1,0.2,0.7]$} & 3 & 3 & 76 & 66 & 8 & 26.4 & $\mathrm{Y}$ & 0.5 \\
\hline 3 & {$[0.1,0.3,0.6]$} & 4 & 4 & 58 & 47 & 10 & 25.9 & $\mathrm{Y}$ & 0.4 \\
\hline 4 & {$[0.1,0.4,0.5]$} & 4 & 3 & 72 & 53 & 9 & 32.9 & $\mathrm{Y}$ & 0.5 \\
\hline 5 & {$[0.1,0.5,0.4]$} & 4 & 4 & 58 & 61 & 10 & 40.3 & $\mathrm{Y}$ & 0.4 \\
\hline 6 & {$[0.1,0.6,0.3]$} & 4 & 4 & 53 & 48 & 11 & 37.4 & $\mathrm{Y}$ & 0.5 \\
\hline 7 & {$[0.1,0.7,0.2]$} & 4 & 4 & 63 & 61 & 11 & 51.2 & $\mathrm{Y}$ & 0.5 \\
\hline 8 & {$[0.1,0.8,0.1]$} & 4 & 4 & 65 & 66 & 9 & 60.2 & $\mathrm{Y}$ & 0.4 \\
\hline 9 & {$[0.2,0.1,0.7]$} & 4 & 4 & 57 & 51 & 9 & 22.8 & $\mathrm{Y}$ & 0.7 \\
\hline 10 & {$[0.2,0.2,0.6]$} & 3 & 4 & 55 & 41 & 9 & 24.6 & $\mathrm{Y}$ & 0.6 \\
\hline 11 & {$[0.2,0.3,0.5]$} & 3 & 4 & 61 & 44 & 10 & 30.4 & $\mathrm{Y}$ & 0.4 \\
\hline 12 & {$[0.2,0.4,0.4]$} & 4 & 4 & 53 & 64 & 9 & 39.8 & $\mathrm{Y}$ & 0.4 \\
\hline 13 & {$[0.2,0.5,0.3]$} & 4 & 4 & 57 & 62 & 11 & 45.7 & $\mathrm{Y}$ & 0.5 \\
\hline 14 & {$[0.2,0.6,0.2]$} & 3 & 4 & 58 & 49 & 10 & 43 & $\mathrm{Y}$ & 0.6 \\
\hline 15 & {$[0.2,0.7,0.1]$} & 4 & 4 & 65 & 58 & 9 & 54.5 & $\mathrm{Y}$ & 0.6 \\
\hline 16 & {$[0.3,0.1,0.6]$} & 3 & 3 & 80 & 50 & 9 & 34.4 & $\mathrm{Y}$ & 0.5 \\
\hline 17 & {$[0.3,0.2,0.5]$} & 3 & 3 & 67 & 53 & 9 & 35.2 & $\mathrm{Y}$ & 0.7 \\
\hline 18 & {$[0.3,0.3,0.4]$} & 3 & 3 & 52 & 36 & 7 & 29.2 & $\mathrm{Y}$ & 0.5 \\
\hline 19 & {$[0.3,0.4,0.3]$} & 4 & 4 & 75 & 47 & 10 & 44.3 & $\mathrm{Y}$ & 0.5 \\
\hline 20 & {$[0.3,0.5,0.2]$} & 4 & 3 & 64 & 47 & 8 & 44.3 & $\mathrm{Y}$ & 0.4 \\
\hline 21 & {$[0.3,0.6,0.1]$} & 4 & 4 & 61 & 69 & 10 & 60.7 & $\mathrm{Y}$ & 0.4 \\
\hline 22 & {$[0.4,0.1,0.5]$} & 4 & 4 & 64 & 48 & 11 & 35.9 & $\mathrm{Y}$ & 0.5 \\
\hline 23 & {$[0.4,0.2,0.4]$} & 4 & 4 & 57 & 51 & 9 & 36.6 & $\mathrm{Y}$ & 0.4 \\
\hline 24 & {$[0.4,0.3,0.3]$} & 4 & 3 & 69 & 50 & 7 & 44.7 & $\mathrm{Y}$ & 0.4 \\
\hline 25 & {$[0.4,0.4,0.2]$} & 4 & 4 & 60 & 42 & 10 & 42.8 & $\mathrm{Y}$ & 0.5 \\
\hline 26 & {$[0.4,0.5,0.1]$} & 3 & 3 & 69 & 77 & 7 & 66.8 & $\mathrm{Y}$ & 0.4 \\
\hline 27 & {$[0.5,0.1,0.4]$} & 3 & 3 & 62 & 44 & 9 & 39 & $\mathrm{Y}$ & 0.4 \\
\hline 28 & {$[0.5,0.2,0.3]$} & 4 & 4 & 69 & 45 & 11 & 46.8 & $\mathrm{Y}$ & 0.4 \\
\hline 29 & {$[0.5,0.3,0.2]$} & 3 & 4 & 74 & 73 & 8 & 60.5 & $\mathrm{Y}$ & 0.5 \\
\hline 30 & {$[0.5,0.4,0.1]$} & 4 & 4 & 67 & 56 & 11 & 57 & $\mathrm{Y}$ & 0.4 \\
\hline 31 & {$[0.6,0.1,0.3]$} & 4 & 4 & 69 & 41 & 10 & 48.5 & $\mathrm{Y}$ & 0.4 \\
\hline 32 & {$[0.6,0.2,0.2]$} & 4 & 3 & 70 & 50 & 10 & 54 & $\mathrm{Y}$ & 0.4 \\
\hline 33 & {$[0.6,0.3,0.1]$} & 4 & 4 & 72 & 44 & 9 & 57.3 & $\mathrm{Y}$ & 0.4 \\
\hline 34 & {$[0.7,0.1,0.2]$} & 3 & 3 & 71 & 73 & 8 & 58.6 & $\mathrm{Y}$ & 0.4 \\
\hline 35 & {$[0.7,0.2,0.1]$} & 3 & 4 & 61 & 73 & 8 & 58.1 & $\mathrm{Y}$ & 0.4 \\
\hline 36 & {$[0.8,0.1,0.1]$} & 4 & 4 & 80 & 57 & 11 & 70.8 & $\mathrm{Y}$ & 0.5 \\
\hline
\end{tabular}

chain. Cross-docking approach can help to increase the coordination efficiency and reduce the transshipment time and operations cost. This is especially important in the quick response-based fashion supply chain with multiproduct and small-batch distribution. In cross-docking operations, a cross-docking center separates the suppliers and customers into two groups, which can be modeled as two VRP models, respectively. However, it is obvious that a system-wide optimal operations performance in the cross-docking center requires coordinating these two models so as to obtain the system-wide optimal strategy. In this paper, we have proposed a truck routing model based on two coordinated VRP models with respect to the commonly observed crossdocking center operations in the fashion supply chain. A 0/1 integer linear programming model has been built for solving it. Furthermore, our numerical analysis illustrates that our proposed optimization problem, with a realistic problem size, can be solved by commonly available optimization software with a very promising performance. However, notice that the VRP is itself an NP-hard problem, and our proposed model focuses on coordinating two VRP models and hence is much more complex in computation. Thus, when the scale of served suppliers and customers is increased or the number of nodes in routes is increased, it will be very difficult to obtain the optimal solution within a few seconds or minutes. Therefore, like many prior studies on VRP in the literature, heuristicbased approaches will play an important role to tackle the problem under such situations. In addition, this study does 
TABLE 6: Performance evaluation of different problem scales.

\begin{tabular}{lcccccc}
\hline No. & INN & IVN & ONN & OVN & Time (s) & Is it optimal? \\
\hline 1 & 2 & 1 & 2 & 1 & 0.1 & $\mathrm{Y}$ \\
2 & 4 & 2 & 4 & 2 & 0.1 & $\mathrm{Y}$ \\
3 & 6 & 3 & 6 & 3 & 0.3 & $\mathrm{Y}$ \\
4 & 8 & 4 & 8 & 4 & 0.5 & $\mathrm{Y}$ \\
5 & 10 & 5 & 10 & 5 & 6.4 & $\mathrm{Y}$ \\
6 & 12 & 6 & 12 & 6 & 20.6 & $\mathrm{Y}$ \\
7 & 14 & 7 & 14 & 7 & 73.2 & $\mathrm{Y}$ \\
8 & 16 & 8 & 16 & 8 & 175.4 & $\mathrm{Y}$ \\
9 & 18 & 9 & 18 & 9 & 312.4 & $\mathrm{Y}$ \\
10 & 20 & 10 & 20 & 10 & 911.2 & $\mathrm{Y}$ \\
11 & 22 & 11 & 22 & 11 & 1862.9 & $\mathrm{Y}$ \\
12 & 24 & 12 & 24 & 12 & 3982.7 & $\mathrm{Y}$ \\
\hline
\end{tabular}

not take into account other practical elements, such as stochastic nature of various model parameters. Considering that some of these elements will make the problem more complex and challenging, we leave them as the topics to be pursued in future research.

\section{Acknowledgments}

The paper was supported partially by the National Natural Science Foundation of China (71101088, 71101028, and 71171129), the National Social Science Foundation of China (11\&ZD169), the Shanghai Municipal Natural Science Foundation (10ZR1413200, 10190502500, 11510501900, and 12ZR1412800), the China Postdoctoral Science Foundation (2011M500077 and 2012T50442), the Science Foundation of Ministry of Education of China (10YJC630087), the Doctoral Fund of Ministry of Education of China (20113121120002), the Program for Excellent Talents and the Program for Innovative Research Team in UIBE. The authors claim that none of them has any financial relation with the commercial identities mentioned in the paper that might lead to conflict of interests.

\section{References}

[1] W.-S. Lo, T.-P. Hong, and R. Jeng, "A framework of E-SCM multi-agent systems in the fashion industry," International Journal of Production Economics, vol. 114, no. 2, pp. 594-614, 2008.

[2] C. H. Chiu, T. M. Choi, H. T. Yeung, and Y. Zhao, "Sales rebate contracts in fashion supply chains," Mathematical Problems in Engineering, vol. 2012, Article ID 908408, 19 pages, 2012.

[3] J. Van Belle, P. Valckenaers, and D. Cattrysse, "Cross-docking: state of the art," Omega, vol. 40, no. 6, pp. 827-846, 2012.

[4] I. F. A. Vis and K. J. Roodbergen, "Positioning of goods in a cross-docking environment," Computers and Industrial Engineering, vol. 54, no. 3, pp. 677-689, 2008.

[5] B. Schaffer, "Cross docking can increase efficiency," Automatic I.D. News, vol. 14, no. 8, pp. 34-37, 1998.

[6] T.-M. Choi, "Quick response in fashion supply chains with dual information updating," Journal of Industrial and Management Optimization, vol. 2, no. 3, pp. 255-268, 2006.
[7] T.-M. Choi, "Pre-season stocking and pricing decisions for fashion retailers with multiple information updating," International Journal of Production Economics, vol. 106, no. 1, pp. 146-170, 2007.

[8] T. M. Choi and S. Sethi, "Innovative quick response programs: a review," International Journal of Production Economics, vol. 127, no. 1, pp. 1-12, 2010.

[9] M. Bertolini, M. Bevilacqua, E. Bottani, and A. Rizzi, "Requirements of an ERP enterprise modeller for optimally managing the fashion industry supply chain," Journal of Enterprise Information Management, vol. 17, no. 3, pp. 180-190, 2004.

[10] J. L. Salmeron and J. M. Hurtado, "Modelling the reasons to establish B2C in the fashion industry," Technovation, vol. 26, no. 7, pp. 865-872, 2006.

[11] S. Guercini and A. Runfola, "Business networks and retail internationalization: a case analysis in the fashion industry," Industrial Marketing Management, vol. 39, no. 6, pp. 908-916, 2010.

[12] M. Christopher, R. Lowson, and H. Peck, "Creating agile supply chain in the fashion industry," International Journal of Retail and Distribution Management, vol. 32, no. 8, pp. 367-376, 2004.

[13] A. Şen, "The US fashion industry: a supply chain review," International Journal of Production Economics, vol. 114, no. 2, pp. 571-593, 2008.

[14] M. Bruce, L. Daly, and N. Towers, "Lean or agile: a solution for supply chain management in the textiles and clothing industry?" International Journal of Operations and Production Management, vol. 24, no. 1-2, pp. 151-170, 2004.

[15] G. Birtwistle, N. Siddiqui, and S. S. Fiorito, "Quick response perceptions of UK fashion retailers," International Journal of Retail and Distribution Management, vol. 31, no. 2, pp. 118-128, 2003.

[16] A. Ross and V. Jayaraman, "An evaluation of new heuristics for the location of cross-docks distribution centers in supply chain network design," Computers and Industrial Engineering, vol. 55, no. 1, pp. 64-79, 2008.

[17] C. S. Sung and W. Yang, "An exact algorithm for a crossdocking supply chain network design problem," Journal of the Operational Research Society, vol. 59, no. 1, pp. 119-136, 2008.

[18] S. S. Heragu, L. Du, R. J. Mantel, and P. C. Schuur, "Mathematical model for warehouse design and product allocation," International Journal of Production Research, vol. 43, no. 2, pp. 327-338, 2005.

[19] G. Alpan, R. Larbi, and B. Penz, "A bounded dynamic programming approach to schedule operations in a cross docking platform," Computers and Industrial Engineering, vol. 60, no. 3, pp. 385-396, 2011.

[20] I. F. A. Vis and K. J. Roodbergen, "Layout and control policies for cross docking operations," Computers and Industrial Engineering, vol. 61, no. 4, pp. 911-919, 2011.

[21] R. Soltani and S. J. Sadjadi, "Scheduling trucks in cross-docking systems: a robust meta-heuristics approach," Transportation Research E, vol. 46, no. 5, pp. 650-666, 2010.

[22] N. Boysen, "Truck scheduling at zero-inventory cross docking terminals," Computers and Operations Research, vol. 37, no. 1, pp. 32-41, 2010.

[23] N. Boysen, M. Fliedner, and A. Scholl, "Scheduling inbound and outbound trucks at cross docking terminals," OR Spectrum, vol. 32, no. 1, pp. 135-161, 2009.

[24] B. Vahdani and M. Zandieh, "Scheduling trucks in crossdocking systems: robust meta-heuristics," Computers and Industrial Engineering, vol. 58, no. 1, pp. 12-24, 2010. 
[25] Y. H. Lee, J. W. Jung, and K. M. Lee, "Vehicle routing scheduling for cross-docking in the supply chain," Computers and Industrial Engineering, vol. 51, no. 2, pp. 247-256, 2006.

[26] D. L. McWilliams, "A dynamic load-balancing scheme for the parcel hub-scheduling problem," Computers and Industrial Engineering, vol. 57, no. 3, pp. 958-962, 2009.

[27] Y. Oh, H. Hwang, C. N. Cha, and S. Lee, "A dock-door assignment problem for the Korean mail distribution center," Computers and Industrial Engineering, vol. 51, no. 2, pp. 288296, 2006.

[28] W. Yu and P. J. Egbelu, "Scheduling of inbound and outbound trucks in cross docking systems with temporary storage," European Journal of Operational Research, vol. 184, no. 1, pp. 377-396, 2008.

[29] Y. Cohen and B. Keren, "Trailer to door assignment in a synchronous cross-dock operation," International Journal of Logistics Systems and Management, vol. 5, no. 5, pp. 574-590, 2009.

[30] R. Dondo and J. Cerd?, "A sweep-heuristic based formulation for the vehicle routing problem with cross-docking," Computers and Chemical Engineering, vol. 48, no. 10, pp. 293-311, 2013.

[31] G. Alpan, A. L. Ladier, R. Larbi, and B. Penz, "Heuristic solutions for transshipment problems in a multiple door cross docking warehouse," Computers and Industrial Engineering, vol. 61, no. 2, pp. 402-408, 2011.

[32] M. Wen, J. Larsen, J. Clausen, J. F. Cordeau, and G. Laporte, "Vehicle routing with cross-docking," Journal of the Operational Research Society, vol. 60, no. 12, pp. 1708-1718, 2009.

[33] T. W. Liao, P. J. Egbelu, and P. C. Chang, "Two hybrid differential evolution algorithms for optimal inbound and outbound truck sequencing in cross docking operations," Applied Soft Computing, vol. 12, no. 11, pp. 3683-3697, 2012.

[34] B. Eksioglu, A. V. Vural, and A. Reisman, "The vehicle routing problem: a taxonomic review," Computers and Industrial Engineering, vol. 57, no. 4, pp. 1472-1483, 2009.

[35] V. Pillac, M. Gendreau, C. Guéret, and A. L. Medaglia, "A review of dynamic vehicle routing problems," European Journal of Operational Research, vol. 225, no. 1, pp. 1-11, 2013.

[36] M. Drexl, "Synchronization in vehicle routing-a survey of vrps with multiple synchronization constraints," Transportation Science, vol. 46, no. 3, pp. 297-316, 2012.

[37] Dash Optimization, “Xpress-IVE," 2012, http://www.fico.com/ en/Products/DMTools/Pages/FICO-Xpress-OptimizationSuite.aspx. 


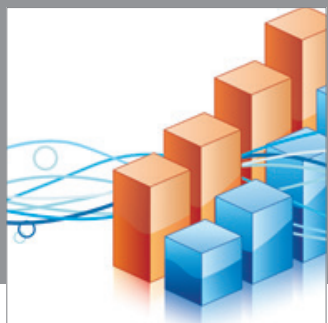

Advances in

Operations Research

mansans

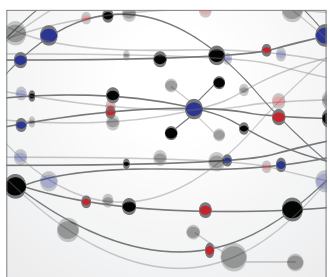

The Scientific World Journal
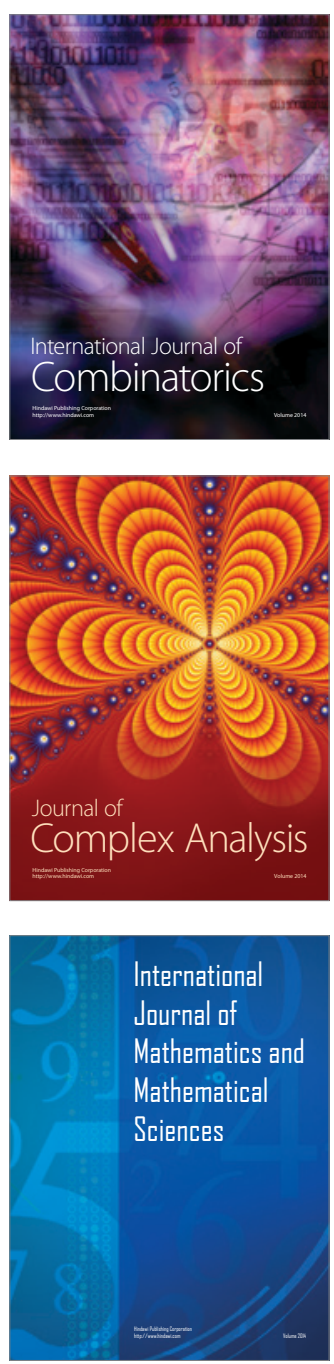
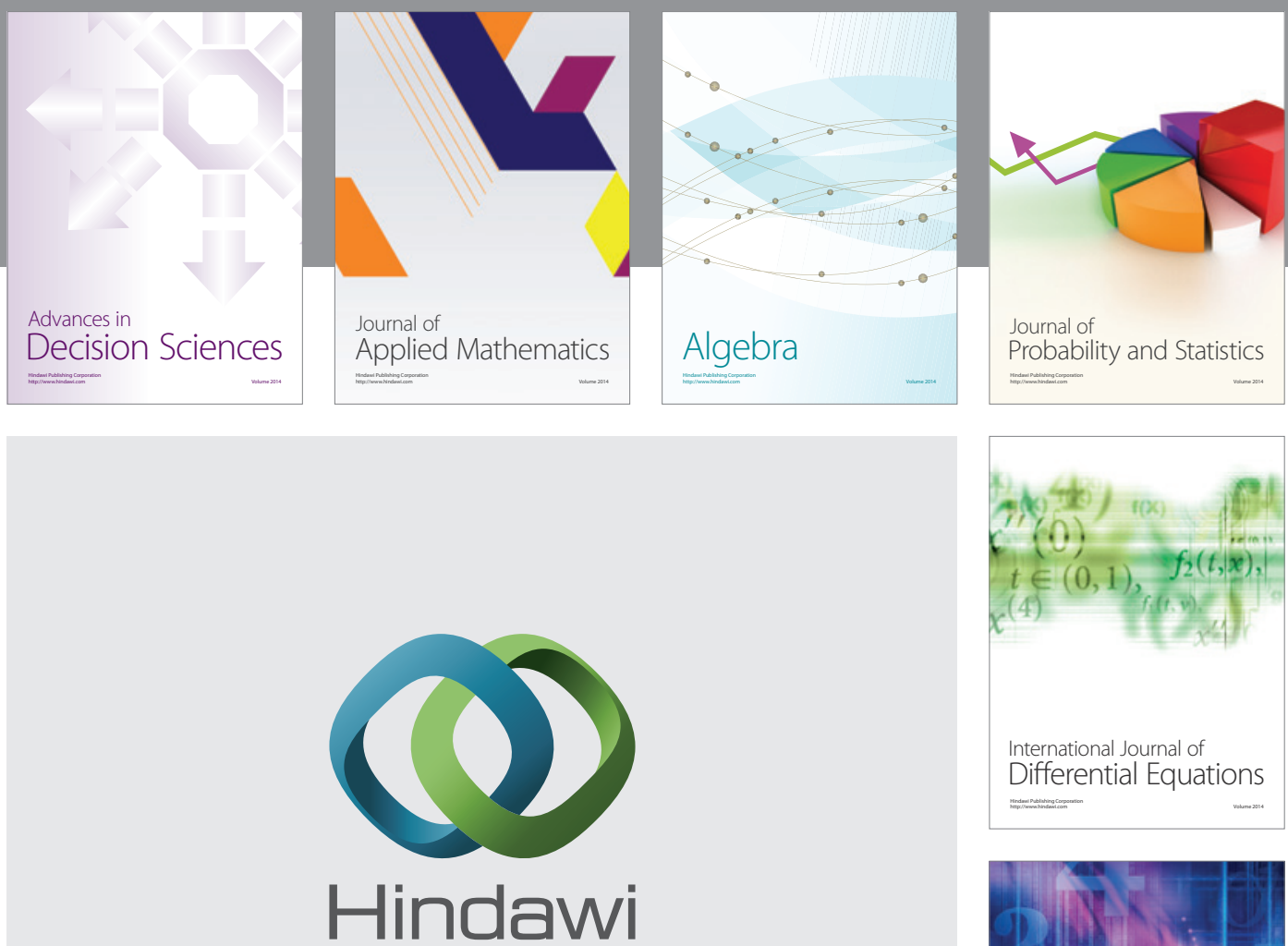

Submit your manuscripts at http://www.hindawi.com
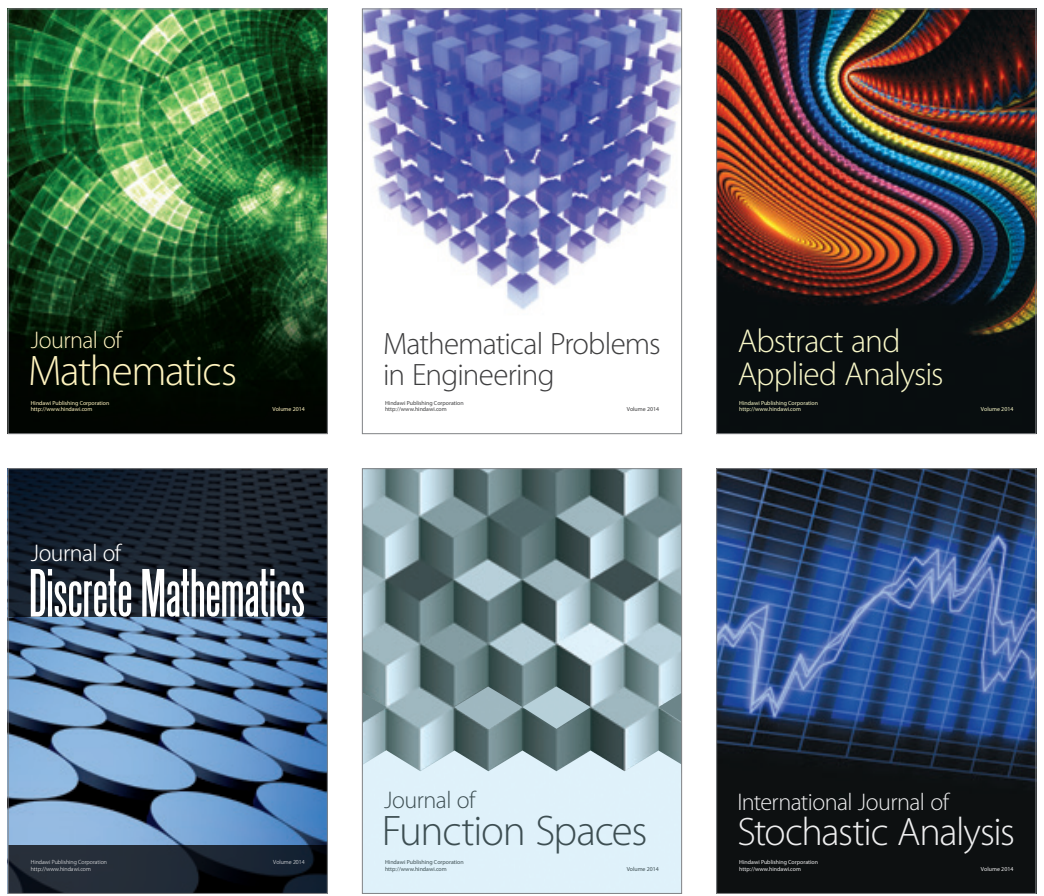

Journal of

Function Spaces

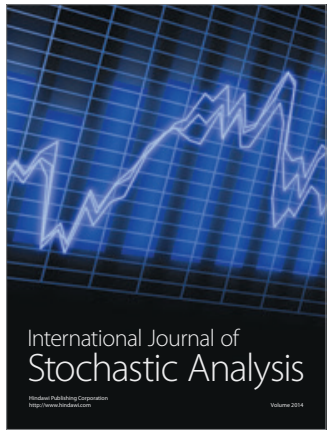

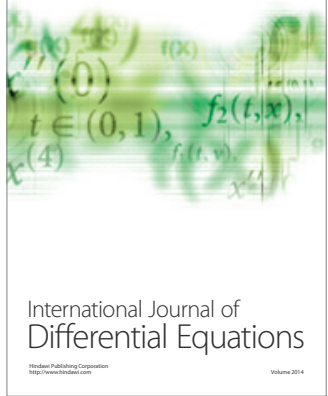
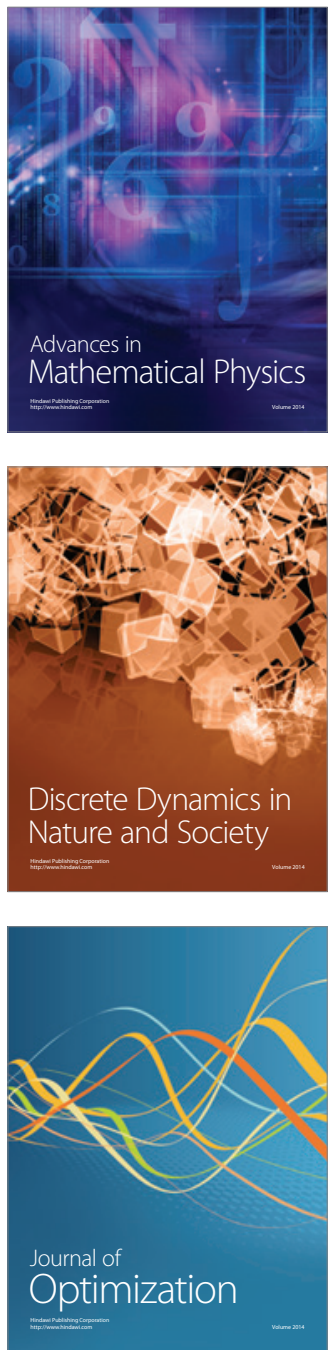